\title{
Screen Printed PZT Thick Films Using Composite Film Technology
}

\author{
R. A. DOREY, ${ }^{\text {a }}$ R. W. WHATMORE, ${ }^{\text {a }}$ S. P. BEEBY,,${ }^{\text {R }}$. N. TORAH, ${ }^{\text {b }}$ \\ and N. M. WHITE ${ }^{\mathrm{b}}$ \\ ${ }^{a}$ Nanotechnology Group, School of Industrial and Manufacturing Science, \\ Cranfield University, Cranfield, Bedfordshire, MK43 OAL, UK \\ ${ }^{\mathrm{b}}$ Department of Electronics and Computer Science, University of Southampton, \\ Southampton, SO17 1BJ, UK
}

(Received May 19, 2003)

A spin coating composite sol gel technique for producing lead zirconate titanate (PZT) thick films has been modified for use with screen printing techniques. The resulting screen printing technique can be used to produce $10 \mu \mathrm{m}$ thick films in a single print. The resultant films are porous but the density can be increased through the use of repeated sol infiltration/pyrolysis treatments to yield a high density film. When fired at $710^{\circ} \mathrm{C}$ the composite screen printed films have dielectric and piezoelectric properties comparable to, or exceeding, those of films produced using a 'conventional' powder/glass frit/oil ink and fired at $890^{\circ} \mathrm{C}$.

Keywords: Lead zirconate titanate; screen printing; composite; sol gel

\section{INTRODUCTION}

Lead zirconate titanate (PZT) ceramics are of great interest for use in micro electromechanical systems (MEMS) due to the high piezoelectric response that they exhibit. To realise Micro-Electro-Mechanical Systems (MEMS) capable of producing large deflections and high actuation forces it is necessary to incorporate thick piezoelectric elements [1]. Typical PZT thicknesses in such MEMS devices range from 5-100 $\mu \mathrm{m}$.

Low temperature, thin film technologies, such as sputtering, pulsed laser deposition and sol gel, require excessive deposition times [2] and are limited in the maximum thickness obtainable due to the build-up of internal stresses during deposition which lead to film cracking [3]. Conversely, the use of conventional bulk ceramic processing with subsequent machining and bonding is both time consuming and wasteful of material (a particular 
environmental/health issue as the waste contains high levels of lead). Other less wasteful high temperature processing routes based on sintering of ceramic particles — such as screen printing and electrophoresis - are of limited applicability due to the requirement for refractory substrates [4] which are difficult to integrate into MEMS processing routes [5]. Even at moderate temperatures, where silicon substrates can be used, the increased diffusion kinetics mean that very thick and robust diffusion barrier/electrode systems are required [6, 7]. This is of particular importance in systems containing $\mathrm{Pb}$ (i.e. the majority of piezoelectric applications) as the formation of undesirable liquid phase lead silicates can occur at temperatures greater than $700^{\circ} \mathrm{C}$ [8].

Spin coating composite film (ComFi) technology has successfully been employed in the production of high density, hard doped PZT films up to $30 \mu \mathrm{m}$ thick and at temperatures as low as $710^{\circ} \mathrm{C}$ so minimising the requirements for robust diffusion barrier/electrode systems [9]. The maximum thickness attainable using spin coating ComFi technology is typically limited by the deposition times required. The maximum single layer thickness is governed by the drying strains within the film which give rise to the generation of stresses. To further increase the single layer thickness achievable it is necessary to reduce the drying strains (and hence stresses) by increasing the solids loading of the slurry (powder + sol). However this tends to result in a composite slurry that is too viscous for use with a spin coating technique. Currently ComFi technology operates using the upper limit of powder loading suitable for spin coating where single layer thicknesses of between 2 and $3 \mu \mathrm{m}$ are achievable. Screen printing slurries/inks typically have a far higher solids loading when compared to spin coating slurries. Due to the lower content of volatile components the associated shrinkage strains on drying are much lower. This allows thicker layers to be deposited and decreases the processing times. An added advantage of the screen printing process is the ability to produce patterned structures during the deposition stage so eliminating the etching process that must be used when patterning spin coated films.

The spin coating powder/sol-gel composite slurry has been modified through increased powder loading to produce an ink for use with screen printing technology for the deposition of PZT thick films. Thick $(10 \mu \mathrm{m})$ PZT films have been produced by screen printing powder/sol-gel slurries onto silicon wafers with a $\mathrm{ZrO}_{2} / \mathrm{Ti} / \mathrm{Pt}$ electrode structure. The resultant dielectric and piezoelectric properties of the films have been measured and compared to those of films produced using a conventional powder/frit/oil screen printing ink. 


\section{EXPERIMENTAL PROCEDURE}

The PZT producing sol was synthesised from lead acetate trihydrate, $\mathrm{Zr}$ isopropoxide, Ti propoxide, niobium ethoxide, antimony ethoxide, manganese acetate and 2-Methoxyethanol [7]. The composite slurry was then produced by ball milling the PZT producing sol, PZT powder ( $3 \mathrm{~g}$ powder $/ \mathrm{ml}$ solferroperm PZ26), dispersant (2 wt\% relative to the PZT powder-Kenrich KR55) and sintering aid (4.7 wt $\%$ relative to the PZT powder - $0.2 \mathrm{CuO}_{2}$ $0.8 \mathrm{PbO}$ ) under nitrogen for 24 hours.

$10 \mu \mathrm{m}$ PZT films were produced by screen printing the composite slurry onto a silicon wafer coated with $40 \mathrm{~nm} \mathrm{ZrO}_{2}, 8 \mathrm{~nm}$ Ti and $200 \mathrm{~nm} \mathrm{Pt}$ $\left(\mathrm{Si} / \mathrm{ZrO}_{2} / \mathrm{Ti} / \mathrm{Pt}\right)$. The screen printed film was then dried at $200^{\circ} \mathrm{C}$ and pyrolysed at $450^{\circ} \mathrm{C}$ for 1 minute. The porous screen printed films were then infiltrated with sol and spun at $2000 \mathrm{rpm}$, dried and pyrolysed in order to increase the density of the films. This was repeated a variety of times for different films to ascertain the effect of such a treatment on the final electrical properties. Once the required number of infiltration/pyrolysis treatments had been carried out the films were sintered at $710^{\circ} \mathrm{C}$ for 30 minuted in an air atmosphere to further densify the films and develop the perovskite structure.

For comparison purposes $10 \mu \mathrm{m}$ thick PZT films were also produced using a conventional screen printing ink consisting of the same PZT powder and $10 \mathrm{wt} \%$ glass frit dispersed in a pine oil carrier medium. The films were fired at $750^{\circ} \mathrm{C}$ and $890^{\circ} \mathrm{C}$.

The crystallinity of films were assessed using X-ray diffraction (Siemens D-5005) prior to the deposition of circular $\mathrm{Cr} / \mathrm{Au}$ top electrode structures by evaporation. Contact was made to the back electrode by removing a small section of the PZT film though mechanical abrasion. Capacitance and loss measurements were made using a Wayne Kerr 6425 analyser at $1 \mathrm{kHz}$. The thicknesses of the films were determined from optical and scanning electron microscope (SEM) observations. Values of thickness and capacitance were then used to calculate the relative permittivity of the thick films. The films were then poled at $130^{\circ} \mathrm{C}$ for 5 minutes using a field of $8 \mathrm{~V} / \mu \mathrm{m}$. Following poling the piezoelectric coefficients $\mathrm{d}_{33, f}$ and $\mathrm{e}_{31, f}$ were measured using a modified piezometer (TakeControl PM25) [10].

\section{RESULTS AND DISCUSSION}

\section{Crystal and Micro-Structures}

Figure 1 shows the $\mathrm{X}$-ray diffraction pattern for the composite screen printed film together with those of the bulk ceramic and a thick film produced using 


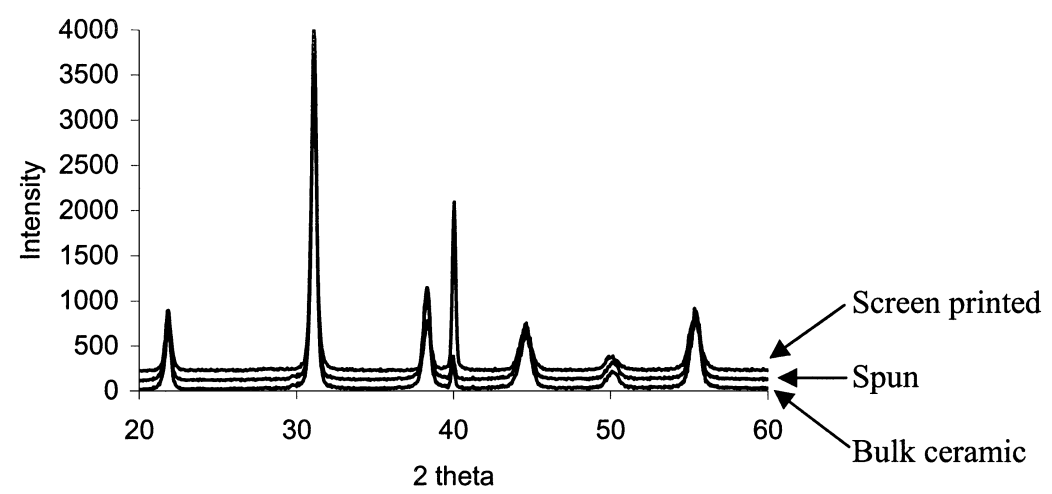

FIGURE 1 X-ray diffraction pattern for screen printed and spun composite films and PZ26 bulk ceramic.

the spin coating composite film technique. It can be seen that the screen printed film exhibits the same random perovskite structure as the bulk ceramic and spin coated thick film. A cross sectional fracture surface of the screen printed films with 6 repeated sol infiltration/pyrolysis treatments is shown in Fig. 2. It can be seen that the resultant film has a high density and a uniform thickness. There is no evidence of density gradients within the structure indicating that the sol is able to completely infiltrate the structure and that the subsequent drying/pryolysis treatment results in a uniform change in density.

Examination of the PZT/Si interface shows that there are no interfacial reactions occurring at the processing temperature of $710^{\circ} \mathrm{C}$. This due to a combination of the low processing temperature and the presence of the $40 \mathrm{~nm}$ $\mathrm{ZrO}_{2}$ diffusion barrier.

\section{Electrical Properties}

The relative permittivity of the composite screen printed thick films is shown as a function of sol infiltration/pyrolysis treatments in Fig. 3. The relative permittivity of the films can be seen to increase from 300 to approximately 600 with increasing numbers of densification treatments. This is a direct consequence of increasing the density of the films. The dielectric loss of the material is independent of the change in density and has a value of approximately $1.5 \%$.

Figure 4 shows the variation in the $\mathrm{d}_{33, f}$ piezoelectric coefficient of the thick films as a function of sol infiltration/pyrolysis. The initial low 


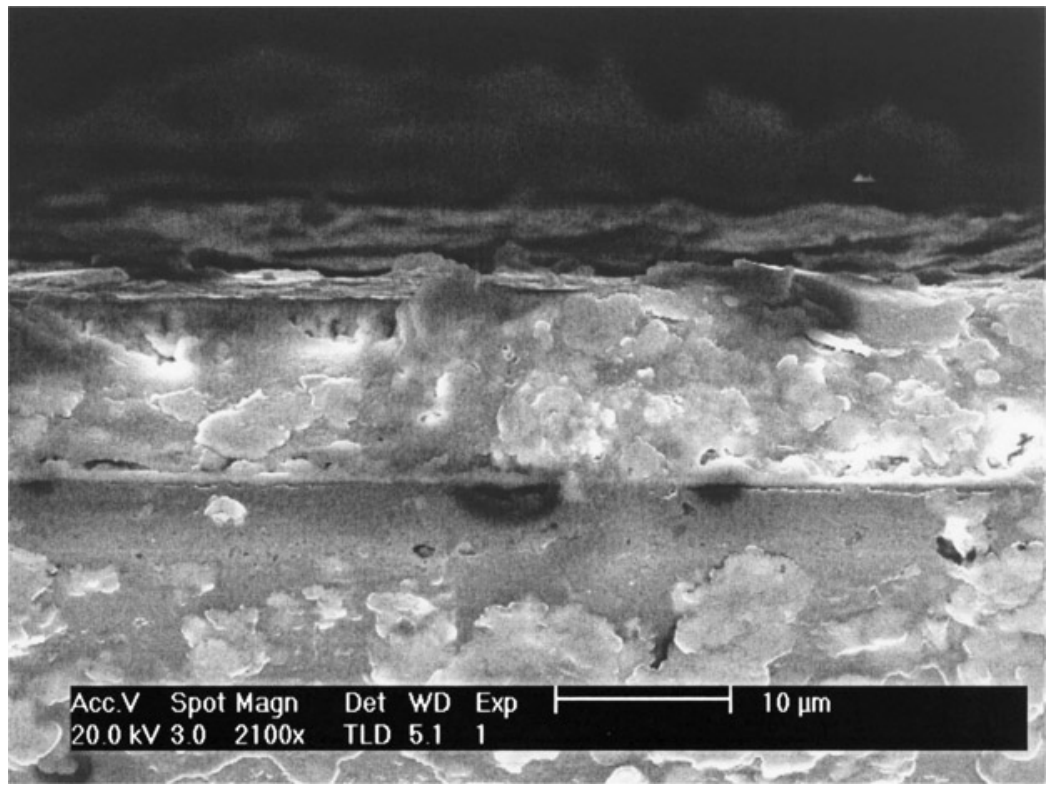

FIGURE 2 Scanning electron microscope photomicrograph of a fracture cross section of a screen printed composite sol gel film treated to 6 repeated sol infiltration/pyrolysis treatments.

piezoelectric coefficients have previously been attributed to deformation modes of the very low density of the films [7]. The $\mathrm{e}_{31, f}$ piezoelectric coefficient was found to be approximately $-0.3 \mathrm{C} / \mathrm{m}^{2}$ and independent of the level of sol infiltration/pyrolysis treatments. This value is relatively low in comparison to values obtained from spin coated composite thick films which exhibit values of $\mathrm{e}_{31, f}$ as high as $-8 \mathrm{C} / \mathrm{m}^{2}$. Analysis of the surface of the screen printed films shows the presence of large cracks that could act as 'spacers' between islands of active material. These spacers would prevent the bending motion from being transferred though the whole structure and so result in an artificially low $\mathrm{e}_{31, f}$ piezoelectric coefficient. Current work is examining the cause of these cracks in an attempt to increase the value of $\mathrm{e}_{31, f}$.

\section{Comparison of Printing Inks}

Table I shows a comparison between the electrical properties of the films produced using the composite sol gel route and those produced using a 


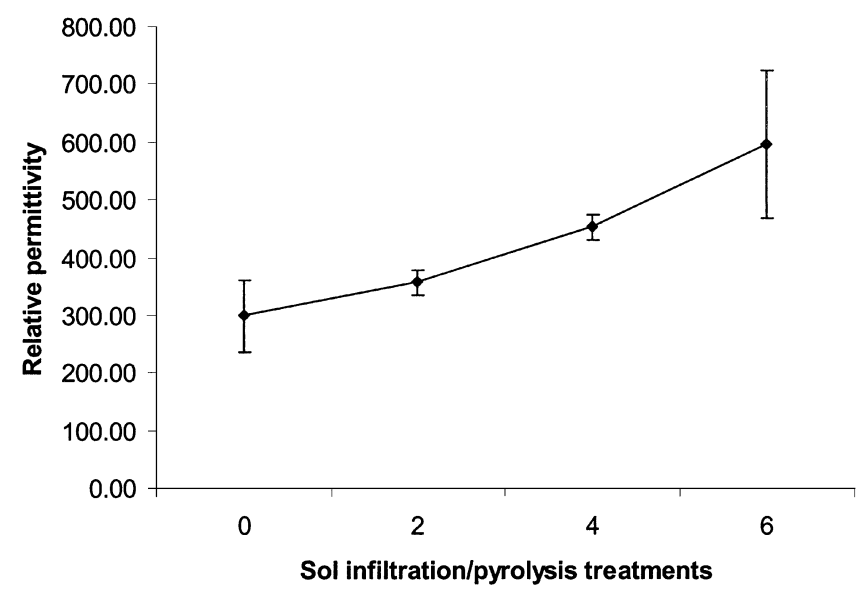

FIGURE 3 Relative permittivity of screen printed composite sol gel films as a function of sol infiltration/pyrolysis treatments.

conventional screen printing ink. It can be seen that for films produced at similar temperatures the composite sol gel route yields films with much higher electrical properties. This is in part due to the presence of the small sized sol gel derived PZT crystals which have a significantly higher thermodynamic driving force for sintering.

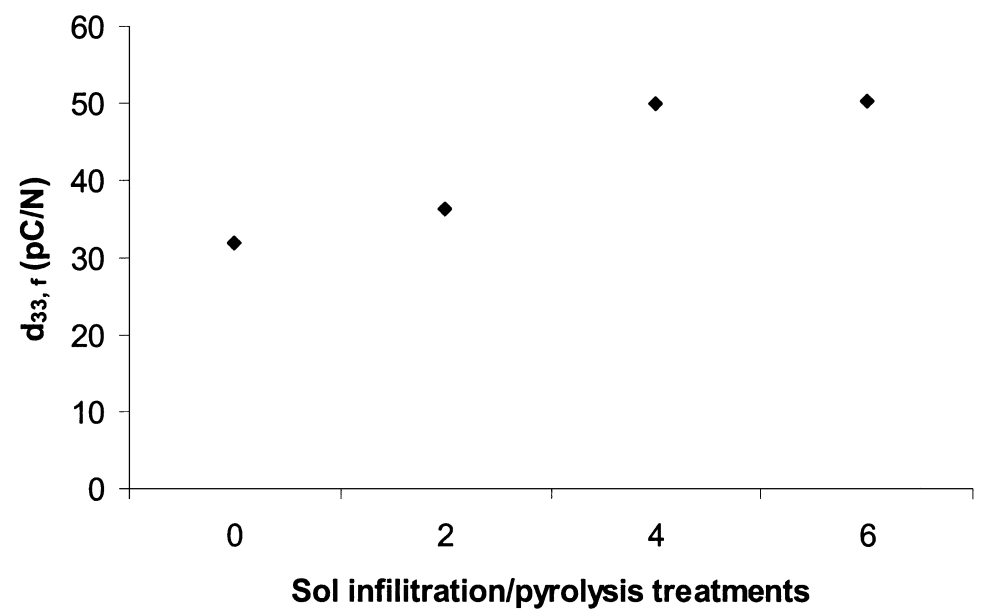

FIGURE $4 \mathrm{~d}_{33, f}$ piezoelectric coefficient of screen printed composite sol gel films as a function of sol infiltration/pyrolysis treatments. 
TABLE I Comparison of electrical properties of films produced using the composite sol gel ink and those produced using the conventional screen printing ink

\begin{tabular}{lccc}
\hline & Composite sol gel & \multicolumn{2}{c}{ Conventional ink } \\
\hline Max processing T & $710^{\circ} \mathrm{C}$ & $750^{\circ} \mathrm{C}$ & $890^{\circ} \mathrm{C}$ \\
Relative permittivity & 600 & 290 & 615 \\
$\mathrm{~d}_{33, f \text { piezo. coef. }}$ & $50 \mathrm{pC} / \mathrm{N}$ & $20 \mathrm{pC} / \mathrm{N}$ & $20 \mathrm{pC} / \mathrm{N}$ \\
$\mathrm{e}_{31, f}$ piezo. coef. & $-0.3 \mathrm{C} / \mathrm{m}^{2}$ & $-0.2 \mathrm{C} / \mathrm{m}^{2}$ & $-0.7 \mathrm{C} / \mathrm{m}^{2}$ \\
\hline
\end{tabular}

When the processing temperature of the conventional ink films is increased to $890^{\circ} \mathrm{C}$ the electrical properties of the resultant films are increased such that the relative permittivity is comparable to that of the composite sol gel film processed at $710^{\circ} \mathrm{C}$. This is in part due to the presence of the sol gel derived PZT. Initially the sol gel phase increases the density of the 'green' structure so reducing the amount of high temperature densification required. Secondly, the sol gel phase - composed of nanoscale particlessignificantly increases the thermodynamic driving force for sintering. This will lead to a decrease in the processing temperature required to sinter the structure. The addition of the low temperature sintering aid serves the same purpose as the glass frit in the conventional ink (i.e. increases the densification kinetics). However, less sintering is used which will help to maintain the electrical properties.

The $\mathrm{d}_{33, f}$ piezoelectric coefficient is still lower than that of the composite film probably due to the high level of non-piezoelectric glass frit present. The slightly higher $\mathrm{e}_{31, f}$ piezoelectric coefficient can be attributed to combined action of the absence of cracks in the film so raising the value and the presence of the glass frit which lowers the value.

\section{CONCLUSIONS}

$10 \mu \mathrm{m}$ thick films have been produced using a composite sol gel screen printing technique. High density films were obtained following repeated sol infiltration and pyrolysis of the porous screen printed films. A final high temperature sintering stage was then used to fully develop the perovskite phase.

The resultant films exhibit dielectric and piezoelectric properties that are comparable to, or greater than, films produced at $890^{\circ} \mathrm{C}$ using a conventional powder/frit/oil ink. 


\section{REFERENCES}

[1] E. S. Thiele, D. Damjanovic, and N. Setter, J. Am. Ceram. Soc. 84(12), 2863-2868 (2001).

[2] N. Setter, J. Euro. Ceram. Soc. 21, 1279-1293 (2001).

[3] Q. F. Zhou, H. L. W. Chen, and C. L. Choy, Thin Solid Films 375, $95-99$ (2000).

[4] R. Mass, M. Koch, N. R. Harris, N. M. White, and A. G. R. Evans, Materials Letters 31, 109-112 (1997).

[5] D. A. Payne and P. G. Clem, J. Electroceram. 3, 163-172 (1999).

[6] Y. B. Kim, T. S. Kim, K. S. Choi, and D. J. Choi, Intergrated Ferroelectrics 35, 199-208 (2001).

[7] S. P. Beeby, A. Blackburn, and N. M. White, J. Micromech. Microeng. 9, 218-229 (1999).

[8] O. M. Kanunnikova, F. Z. Gilmutdinov, and A. A. Shakov, International Journal of Hydrogen Energy 27, 783-791 (2002).

[9] R. A. Dorey, S. B. Stringfellow, and R. W. Whatmore, J. Euro. Ceram. Soc. 22, 2921-2926, (2002).

[10] J. E. A. Southin, S. A. Wilson, D. Schmitt, and R. W. Whatmore, J. Phys. D: Appl. Phys. 34, 1456-1460 (2001). 